\title{
Top-quark properties at ATLAS with focus on the latest mass and spin correlation measurements
}

\author{
Markus Cristinziani ${ }^{* \dagger}$ \\ On behalf of the ATLAS Collaboration \\ Physikalisches Institut, Universität Bonn, Nussallee 12, 53115 Bonn, Germany. \\ E-mail: cristinz@uni-bonn.de
}

\begin{abstract}
Properties of the top-quark are presented, with emphasis on the most recent ATLAS measurements of the mass and $t \bar{t}$ spin correlations, obtained with proton-proton collision data collected at the Large Hadron Collider. Normalised differential distributions are used in both cases. For the extraction of the top-quark mass, $t \bar{t}+1$-jet single-lepton events are selected from the $20.2 \mathrm{fb}^{-1}$ $8 \mathrm{TeV}$ dataset, and the unfolded distribution at parton level is compared with theoretical predictions to obtain $m_{t}^{\text {pole }}=171.1_{-1.1}^{+1.2} \mathrm{GeV}$ in the pole-mass scheme and $m_{t}\left(m_{t}\right)=162.9_{-1.6}^{+2.4} \mathrm{GeV}$ in the running-mass scheme. For the measurement of spin correlations in $t \bar{t}$ production, dilepton events are selected using $36.1 \mathrm{fb}^{-1} 13 \mathrm{TeV}$ data. The azimuthal opening angle between the two leptons is measured inclusively and as a function of the invariant mass of the $t \bar{t}$ system. The observed degree of spin correlation is significantly higher than predicted by the generators used, but agrees well with the prediction of one of the fixed-order calculations.
\end{abstract}

7th Annual Conference on Large Hadron Collider Physics — LHCP2019

20-25 May, 2019

Puebla, Mexico

\footnotetext{
*Speaker.

${ }^{\dagger}$ Supported by the European Research Council grant ERC-CoG-617185 and by the German Federal Ministry of Education and Research (FSP-103)
} 


\section{Introduction}

The mass of the top quark, the heaviest known elementary particle, is a key parameter of the Standard Model (SM) of particle physics and must be determined experimentally. In the SM, the gauge structure of the interaction of the top quark with other particles establishes a relation between the top-quark, Higgs-boson and $W$-boson masses. A precise determination of these three parameters forms an important consistency check of the SM, and provides information about the evolution of the Higgs quartic coupling, which affects the shape of the Higgs potential and is associated with the stability of the quantum vacuum. Owing to the large mass, the lifetime of the top quark is shorter than the timescale for hadronisation $\left(\sim 10^{-23} \mathrm{~s}\right)$ and is much shorter than the spin decorrelation time $\left(\sim 10^{-21} \mathrm{~s}\right)$. As a result, the spin information of the top quark is transferred directly to its decay products. Top-quark pair production $(t \bar{t})$ in QCD is parity invariant and hence the top quarks are not expected to be polarised in the SM; however, the spins of the top and the anti-top quarks are predicted to be correlated. The determination of $t \bar{t}$ spin correlation is a sensitive test to physics beyond the Standard Model (BSM), since the latter might modify the observed level of correlation.

\section{Measurement of the top-quark pole mass in $t \bar{t}+1$-jet events}

A quantitative statement about the value of a quark mass requires a precise reference to the mass scheme in which the mass is defined. The mass scheme which is used most often in topquark mass measurements is the pole-mass scheme, where the renormalised top-quark mass (the pole mass, $m_{t}^{\text {pole }}$ ) coincides with the pole of the top-quark propagator. From the $t \bar{t}$ production cross section, on other hand, the running top-quark mass in the modified minimal subtraction scheme $(\overline{\mathrm{MS}})$ has been extracted. The two mass schemes can be related precisely, with up to four-loop accuracy.

Direct top-quark mass measurements at hadron colliders, based on the reconstruction of the top-quark decay products and using Monte Carlo (MC) event generators to extract the mass, are frequently interpreted as the pole mass. Recent works estimate that such an interpretation is affected by a $0.5-1 \mathrm{GeV}$ uncertainty due to non-perturbative effects. With direct top-quark mass measurements reaching sub-percent precision it becomes important to evaluate uncertainties associated with the interpretation of the measured mass at the same level of accuracy. It is therefore important to extract the top-quark mass by comparing data with predictions computed in a well-defined mass scheme. In this case the ambiguity related to the top-quark mass interpretation is avoided, allowing a precise evaluation of the uncertainty associated with the mass scheme chosen. In such measurements the MC event generator is only used to correct distributions obtained from measured data for effects originating from the detector and the modelling of non-perturbative physics.

ATLAS reported measurements of the top-quark mass in both, the pole-mass and $\overline{\mathrm{MS}}$ schemes, taking advantage of the sensitivity to the top-quark mass of the differential cross section of $t \bar{t}$ production in association with at least one energetic jet [1]. The measurement is performed using $20.2 \mathrm{fb}^{-1}$ of $8 \mathrm{TeV} p p$ collisions collected in 2012. The presence of the additional jet enhances the sensitivity to the top-quark mass in comparison with similar observables defined for the $t \bar{t}$ system only. In particular, the observable used to extract the top-quark mass, $\mathcal{R}$, is defined as 
the normalised differential $t \bar{t}+1$-jet cross section as a function of the variable $\rho_{\mathrm{s}}=2 m_{0} / m_{t \bar{t}+1 \text {-jet }}$, $\mathcal{R}\left(m_{t}^{\text {pole }}, \rho_{\mathrm{s}}\right)=\left(1 / \sigma_{t \bar{t}+1-\text { jet }}\right) \cdot \mathrm{d} \sigma_{t \bar{t}+1-\text { jet }} / \mathrm{d} \rho_{\mathrm{s}}$, where $m_{0}$ is a constant fixed to $170 \mathrm{GeV}$ and $m_{t \bar{t}+1-\text { jet }}$ is the invariant mass of the $t \bar{t}+1$-jet system. The normalised differential cross sections are presented at the so-called particle level in which data are only unfolded for detector effects and at the parton level where $\mathcal{R}$ can be directly compared with available fixed-order calculations. The large $8 \mathrm{TeV}$ dataset makes it possible to achieve a high precision in the measurement of the $\mathcal{R}$ distribution, in particular in the region where it is most sensitive to the top-quark mass, ultimately allowing the top-quark mass to be extracted with high accuracy.

The nominal $t \bar{t}$ sample that is used to unfold the data was generated using the PowHEG-hvq package, which is based on next-to-leading-order (NLO) QCD matrix elements. The event selection targets the single lepton $t \bar{t}$ signature with one reconstructed electron or muon and at least five jets, two of which must be $b$-jets. After requirements on the transverse momentum $\left(p_{\mathrm{T}}\right)$, the missing transverse momentum $E_{\mathrm{T}}^{\text {miss }}$ and the $W$-boson transverse mass, about 28000 events are selected, of which $93 \%$ are expected to be $t \bar{t}$ events according to simulation.

The $t \bar{t}+1$-jet system is then reconstructed. Candidates for the hadronically decaying $W$ boson are formed by pairing all jets not tagged as $b$-jets and selecting pairs $i, j$ that satisfy $0.9<m_{W} / m_{i j}<$ 1.25 and $\min \left(p_{\mathrm{T}}^{i}, p_{\mathrm{T}}^{j}\right) \cdot \Delta R_{i j}<90 \mathrm{GeV}$ where $p_{\mathrm{T}}^{i}$ is the transverse momentum of the jet $i, m_{i j}$ is the invariant mass of the jet pair, $\Delta R_{i j}$ their angular distance. The application of these two requirements reduces the multijet and combinatorial backgrounds. The neutrino momentum is reconstructed, up to a twofold ambiguity, by identifying the $E_{\mathrm{T}}^{\text {miss }}$ with its transverse momentum and using the $W$ boson mass constraint to infer its longitudinal momentum. Only events where at least one neutrino candidate exists are considered. If there are two solutions, each of the neutrino candidates is added to the charged lepton, leading to two $W$-boson candidates.

Pairs of hadronic and semileptonic top-quark candidates are formed by combining all the hadronic and leptonic $W$-boson candidates with the two $b$-tagged jets. Among all possible combinations the one selected is that which minimises the absolute difference between the masses of the reconstructed hadronic top $\left(m_{t_{\text {had }}}\right)$ and the semileptonic top $\left(m_{t_{\text {lep }}}\right)$ candidates, divided by their sum, $\left|m_{t_{\text {lep }}}-m_{t_{\text {had }}}\right| /\left(m_{t_{\text {lep }}}+m_{t_{\text {had }}}\right)$. The $t \bar{t}$ candidates must satisfy $m_{t_{\text {lep }}} / m_{t_{\text {had }}}>0.9$. The four-momenta of the jets which are identified with the hadronic decay of the $W$ boson are corrected by the factor $m_{W} / m_{i j}$. Among the jets not used in either top-quark candidate, the leading jet in $p_{\mathrm{T}}$ is taken as the jet produced in association with the top quarks, before their decay. Only events where this extra jet has a transverse momentum larger than $50 \mathrm{GeV}$ are considered. The purity of the sample is more than $90 \%$. The $t \bar{t}+1$-jet system is reconstructed adding the four-vectors corresponding to the $b$-jets, the selected $W$-boson candidates and the additional jet. The inclusive quantity $\rho_{\mathrm{s}}$ is insensitive to ambiguities in the combinatorics and is not affected by an incorrect pairing of $b$-jets with $W$-boson candidates.

The measured $\mathcal{R}$ distribution is unfolded and compared with theoretical predictions at fixed order, allowing the determination of the top-quark mass in a well-defined theoretical framework. In addition, the $\mathcal{R}$ distribution is also presented at particle level, where data are unfolded for detector effects only. This will allow direct comparisons with possible future theoretical calculations which include top-quark decay and hadronisation effects. The unfolding procedure is detailed in the following. First, the detector-level distribution of $\rho_{\mathrm{s}}$ is re-binned to maximise the sensitivity of the observable to the top-quark mass while keeping enough statistics in each bin. This is achieved 
by choosing a fine binning in the region $\rho_{\mathrm{s}} \gtrsim 0.6$, where the observable is most sensitive to the top-quark mass. Second, the predicted background contribution is subtracted and the distribution is normalised to unity. Finally, the distribution is unfolded using iterative Bayesian unfolding.

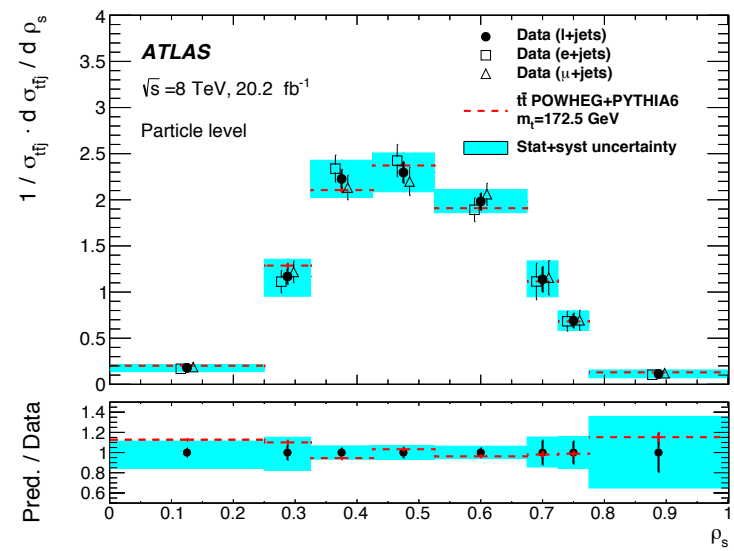

(a)

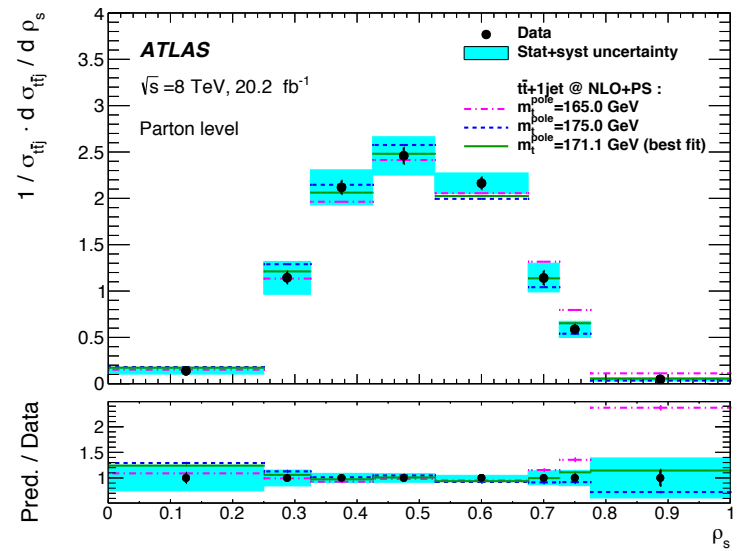

(b)

Figure 1: The normalised differential cross section for $p p \rightarrow t \bar{t}+1$-jet production as a function of $\rho_{\mathrm{s}}$ [1]. (a) The results in the electron and muon channels, and the combination of the two, are shown. The data are unfolded to the particle level and are compared with the prediction from Powheg + Pythia6. (b) The data are unfolded to the parton level. The predictions of the NLO+PS calculation are shown for various top-quark pole mass assumptions.

In Figure 1 the unfolded, normalised differential cross section at particle level is compared with the prediction of the Powheg + Pythia6 generator with the top-quark mass parameter set to $172.5 \mathrm{GeV}$. The distributions obtained from the electron and muon channels separately, unfolded following the nominal procedure, are also presented in the same figure to show their compatibility with the combined result. The same measurement is presented after unfolding to parton level. The result is compared with the prediction for $t \bar{t}+1$-jet production. The fixed-order calculation at NLO accuracy in QCD is interfaced to the parton shower and is labelled as "NLO+PS" in the following. The prediction is shown for two values of the top-quark pole mass, to demonstrate the sensitivity of the observable to the top-quark mass.

The top-quark pole mass is extracted from the parton-level result with an NLO+PS calculation of $t \bar{t}+1$-jet production. In each bin of the distribution a continuous parameterisation $\mathcal{R}_{\mathrm{NLO}+\mathrm{PS}}^{t \bar{t}+1 \text {-jet }}\left(m_{t}^{\mathrm{pole}}\right)$ is obtained by interpolating with a second-order polynomial between different $\mathcal{R}_{\mathrm{NLO}+\mathrm{PS}}^{t \bar{t}+1 \text {-jet }}$ predictions computed at fixed $m_{t}^{\text {pole }}$ values. The fit to the parton-level differential cross section yields the topquark pole mass $m_{t}^{\text {pole }}=171.1 \pm 0.4$ (stat.) \pm 0.9 (syst. $)_{-0.3}^{+0.7}$ (theo.) $\mathrm{GeV}$. The result for the running mass in the $\overline{\mathrm{MS}}$ scheme is $m_{t}\left(m_{t}\right)=162.9 \pm 0.5$ (stat.) \pm 1.0 (syst.) ${ }_{-1.2}^{+2.1}$ (theo.) GeV. Several tests are performed to verify the consistency and robustness of the result, including the choice of $p_{\mathrm{T}}$ cut on the additional jet and of the $\rho_{\mathrm{s}}$ binning. The measured top-quark mass is independent of the assumed top-quark mass in the MC simulation that is used to unfold the data, and is found to be compatible in the electron and muon channels, separately. 


\section{Comparison with other ATLAS top-quark mass measurements}

Figure 2(a) shows a summary of measurements of the top-quark pole mass. The top-quark pole mass result obtained from data unfolded to parton level is compatible with previous measurements. Compared with the result obtained by ATLAS with the same method at $7 \mathrm{TeV}$ the statistical and systematic uncertainties of the new result are reduced by more than a factor of two, yielding a relative uncertainty of $0.7 \%$, constituting the most precise measurement of the top-quark pole mass with $8 \mathrm{TeV}$ data. With $13 \mathrm{TeV}$ data, the CMS collaboration further improved the precision to $0.5 \%$, using differential spectra in the $t \bar{t}$ dilepton channel. The measurements of the top-quark pole mass are competitive with the conventional methods, which are summarised in Figure 2(b). The most recent combination was performed by the ATLAS collaboration [2] using the 7 and $8 \mathrm{TeV} t \bar{t}$ measurements, allowing to reduce the total uncertainty to $0.3 \%$, equalling the measurement of the CMS collaboration that was released three years before. With the analysis of the full Run-2 dataset it is to be expected that both, the conventional methods as well as the extraction of the pole mass will further be improved and attain new levels of precision.

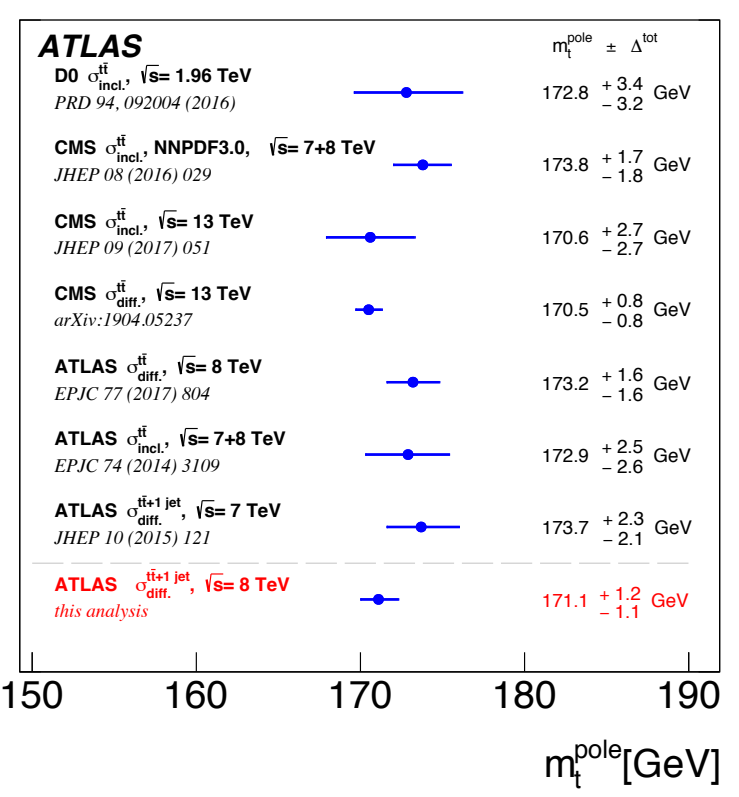

(a)

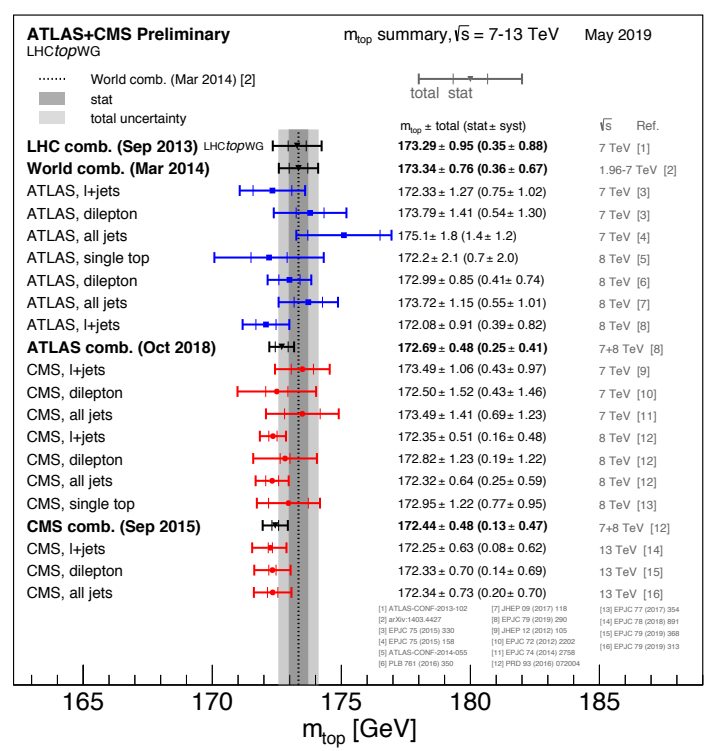

(b)

Figure 2: (a) Summary of top-quark pole mass measurements at the Tevatron and the LHC [1]. (b) Summary of ATLAS and CMS direct $m_{t}$ measurements. The results are compared with the LHC and Tevatron+LHC $m_{t}$ combinations [3].

\section{Spin correlations}

Spin correlations in the $t \bar{t}$ system have been observed experimentally by the ATLAS and CMS collaborations using Run-1 data. A new measurement has been presented by ATLAS [4], using $13 \mathrm{TeV}$ data collected in 2015 and 2016. The spin information of top-quarks can be accessed 
through their decay products. Charged leptons arising from leptonically decaying $W$ bosons carry almost the full spin information of the parent top quark and can be readily identified and reconstructed. Thus, observables to study spin correlation in $t \bar{t}$ events are often based on the angular distributions of the charged leptons in the dilepton channel. Observables used in this measurement are the absolute azimuthal opening angle measured in the transverse plane $(\Delta \phi)$, and the absolute difference between the pseudorapidities $(\Delta \eta)$. Improved MC generators are employed relative to previous spin correlation results to better control the systematic uncertainties. The spin correlation is measured as a function of the invariant mass of the $t \bar{t}$ system, as well as inclusively.

The primary $t \bar{t}$ MC sample used (nominal) was simulated using the NLO PowHEG-Box matrixelement event generator interfaced to PутнIA for the parton shower and fragmentation. An alternative $t \bar{t}$ sample was simulated with the same settings but with the top quarks decayed using MADSPIN and with spin correlations between the $t$ and $\bar{t}$ disabled. This sample was used, along with the nominal sample, as a template in the extraction of spin correlation. Backgrounds with two charged leptons in the final state were simulated using Powheg-Box $(t W)$, Sherpa $(Z+$ jets, diboson) and MADGraPh5_aMC@NLO for other associated top-quark and top-quark pair production. Backgrounds also arise from events containing one prompt lepton from the decay of a $W$ or $Z$ boson and either a non-prompt lepton or a particle misidentified as a lepton. These "fake leptons" were estimated using MC simulations and the result was verified using a same-charge lepton control region in the data; the MC distributions were scaled up by a small amount as a consequence.

Two types of signal events are considered, depending on whether a full reconstruction of the $t \bar{t}$ system is performed, denoted here as inclusive and reconstructed selections. The inclusive selection is used for the $\Delta \phi$ and $\Delta \eta$ differential cross-sections. It is defined by requiring exactly one electron and one muon of opposite electric charge and at least two jets, at least one of which must be $b$-tagged. The reconstructed selection is used for the measurement of $\Delta \phi$ as a function of the $t \bar{t}$ invariant mass. It has a more stringent $b$-tagging requirement of at least two $b$-tagged jets and also requires that at least one solution was found for the reconstruction of the $t \bar{t}$ system. Using the inclusive (reconstructed) selection, 93\% (96\%) of the 177k (76k) selected events are expected to be $t \bar{t}$ events. The data and prediction agree within uncertainties for all kinematic observables studied.

In order to measure spin correlations as a function of the $t \bar{t}$ invariant mass at detector level, the kinematic properties of the event are reconstructed from the identified leptons, jets, and missing transverse momentum. The top quark, top antiquark, and reconstructed $t \bar{t}$ system are built using the neutrino weighting method. The efficiency for $t \bar{t}$ reconstruction is $80 \%$.

Events are corrected for detector effects using two definitions of particles in the generator-level record of the simulation: parton level and particle level. Parton-level objects are taken from the MC simulation history. Top quarks are taken after radiation but before decay whereas leptons are taken before radiation. The measurement corrected to parton level is extrapolated to the full phasespace, where all generated dilepton events are considered. Fiducial requirements are not made on the partonic objects so that the results at parton level can be more easily compared to fixedorder predictions. Particle-level objects are constructed using a procedure intended to correspond as closely as possible to the detector-level object and event selection. Events are selected at the particle level in a fiducial phase-space region with similar requirements to the phase-space region in the detector. 
Background-subtracted data are corrected for detector resolution and acceptance effects using an iterative Bayesian unfolding procedure in order to create distributions at particle (parton) level in a fiducial (full) phase-space. The binning for each observable is chosen in order to minimise the effect of statistical fluctuations in the data as well as in the alternative $t \bar{t}$ samples which are used in the systematic prescription, as well as to account for the experimental resolution. The $\Delta \phi$ distributions are split into four mass regions: $m_{t \bar{t}}<450 \mathrm{GeV} ; 450 \leq m_{t \bar{t}}<550 \mathrm{GeV} ; 550 \leq m_{t \bar{t}}<$ $800 \mathrm{GeV}$; and $m_{t \bar{t}} \geq 800 \mathrm{GeV}$.

The level of spin correlation observed in data is assessed by quantifying it in relation to the amount of correlation expected in the SM. This fraction of SM-like spin correlation $\left(f_{\mathrm{SM}}\right)$ is extracted using hypothesis templates that are fit to the parton-level, unfolded normalised crosssections from data. Two hypotheses are used: dileptonic $t \bar{t}$ events with SM spin correlation (the nominal $t \bar{t}$ sample) and dileptonic events where the effect of spin correlation has been removed (the nominal $t \bar{t}$ sample where the top quarks are decayed using MADSPIN with spin correlations disabled). The extraction of $f_{\mathrm{SM}}$ is performed in five observables: the inclusive $\Delta \phi$ and $\Delta \phi$ in each of the four regions of $m_{t \bar{t}}$. The total number of bins used in the extraction, $N$, depends upon the region of $m_{t \bar{t}}$. Systematic uncertainties on $f_{\mathrm{SM}}$ are determined using MC samples with different sources of systematic uncertainty, and the unfolded spectra are used as pseudo-data. The templates are fit to this pseudo-data and the difference between the systematic $f_{\mathrm{SM}}$ and the nominal (i.e. $f_{\mathrm{SM}}=1$ ) is taken as the systematic uncertainty on $f_{\mathrm{SM}}$ due to that source. The largest sources of systematic uncertainty arise due to the modelling of the $t \bar{t}$ process.

For the inclusive result, the spin correlation extracted from the unfolded data is significantly higher than the SM expectation at a significance of $3.8 \sigma$ without including theoretical uncertainties on the hypothesis templates, and at $3.2 \sigma$ when including these uncertainties. The central $f_{\mathrm{SM}}$ value as a function of $m_{t \bar{t}}$ is found to increase as a function of $m_{t \bar{t}}$, however, the uncertainties on $f_{\mathrm{SM}}$ are much larger than in the inclusive case and none of the results deviate significantly from the SM expectation.

A number of cross-checks were performed to attempt to explain the results in terms of either the limitations of modelling of the $t \bar{t}$ system or by experimental effects not covered by the systematic uncertainties. The generators used in this analysis do not fully include NLO effects in the decays of the top quarks, nor do they directly consider the effects of interference between the initial and final states, but these effects are found not to be relevant for the measurement and do not explain the observed deviation. The effect of the narrow-width approximation (NWA) on the $\Delta \phi$ observable was investigated in an inclusive $t \bar{t}+t W$ dilepton phase-space using the PowHEg-BoxRes bb4l process and compared to the nominal $t \bar{t}+t W$ set-up and no significant differences were observed. It is therefore assumed that, in the $t \bar{t}$ phase-space of this measurement, the NWA in the templates is not a limiting factor and does not explain the observed deviation. Alternative templates for $f_{\mathrm{SM}}$ extraction may be constructed from samples used to evaluate systematic uncertainties. In each case, the no-spin template is derived by scaling the prediction of the alternative model (with spin included) by the ratio of the no-spin and spin templates in the nominal setup. With the exception of the highest $m_{t \bar{t}}$ bin, which has large statistical and systematic uncertainties, the $f_{\mathrm{SM}}$ values remain above 1 for all alternative templates.

The effect of next-to-next-to-leading order (NNLO) corrections in the production was investigated by reweighting the $p_{\mathrm{T}}(t)$ spectra in PowHEG + PyTHIA8 to NNLO fixed-order predictions and 
to observed detector-corrected data spectra. The effect reduced the observed deviation somewhat but was consistent with the scale uncertainties that are already considered in the uncertainties on the hypothesis templates. Fixed-order NNLO predictions recently became available for the observables under study [5]. The results of these predictions are closer to the data than the NLO predictions, but still do not fully describe the observed discrepancy. An alternative differential prediction using a fixed renormalisation and factorisation scale choice of the top mass and performing an expansion in both QCD and electroweak (EW) couplings at NLO, made specifically for these observables [6], is used as a template. This prediction agrees better with the data but has significant scale uncertainties, leading to an $f_{\mathrm{SM}}=1.03 \pm 0.13$, and is consistent both with the result from using the PowHEG + Pythia8 templates and with the SM expectation.

Data is compared with various SM predictions in Figure 3. The disagreement between the data and the NLO predictions from MCFM and PowheG + PythiA8 can be clearly observed. The NNLO fixed-order prediction agrees better with the data but still differs significantly. Finally, the expanded NLO QCD + EW prediction agrees with the data within its large scale uncertainties.

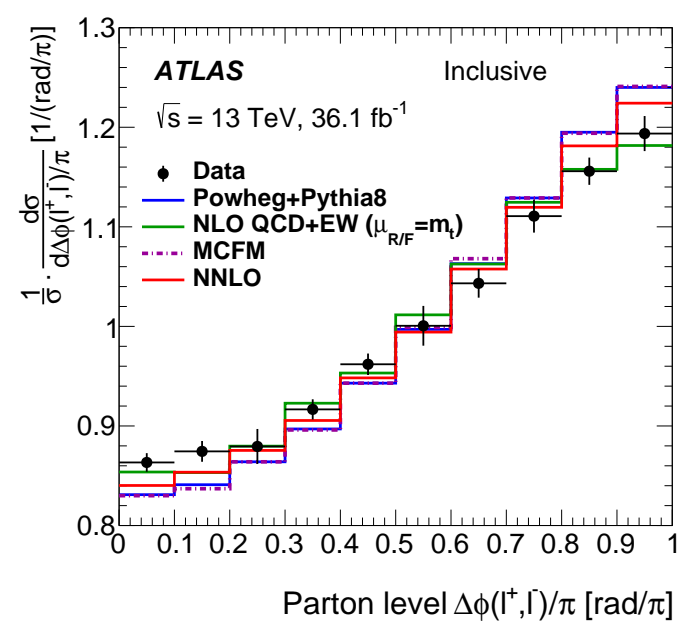

(a)

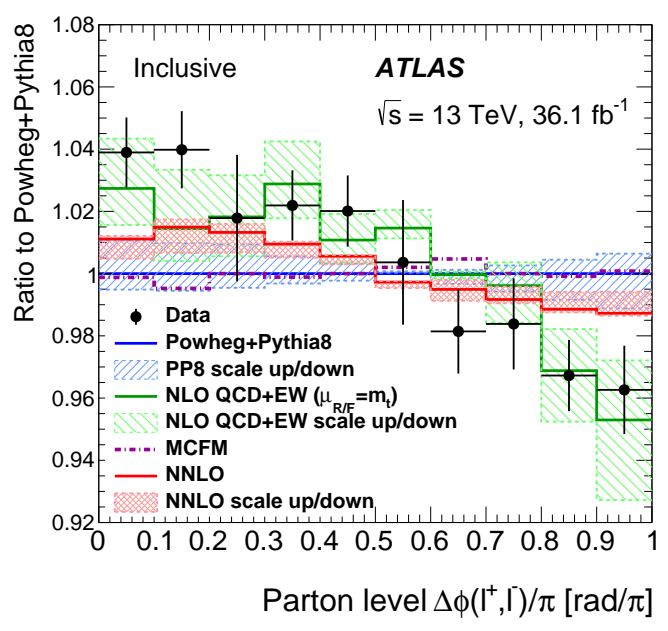

(b)

Figure 3: Comparison of the unfolded $\Delta \phi$ distribution with theoretical predictions for the inclusive selection; (a) normalized cross-section, (b) ratio as compared with PowHEG + PYTHIA8 [4].

\section{Status of other top-quark property measurements and summary}

The most precise top-quark related properties measured by the ATLAS collaboration per LHC run energy are summarised and compared to the corresponding theoretical expectations in Figure 4. The properties are categorised as intrinsic properties of the top quark or as properties of its production or decay. ATLAS top-quark measurements can be conveniently accessed through the TopPublicResults Wiki page, ${ }^{1}$ while the used theoretical predictions are further detailed in Reference [3].

\footnotetext{
${ }^{1}$ https://twiki.cern.ch/twiki/bin/view/AtlasPublic/TopPublicResults
} 


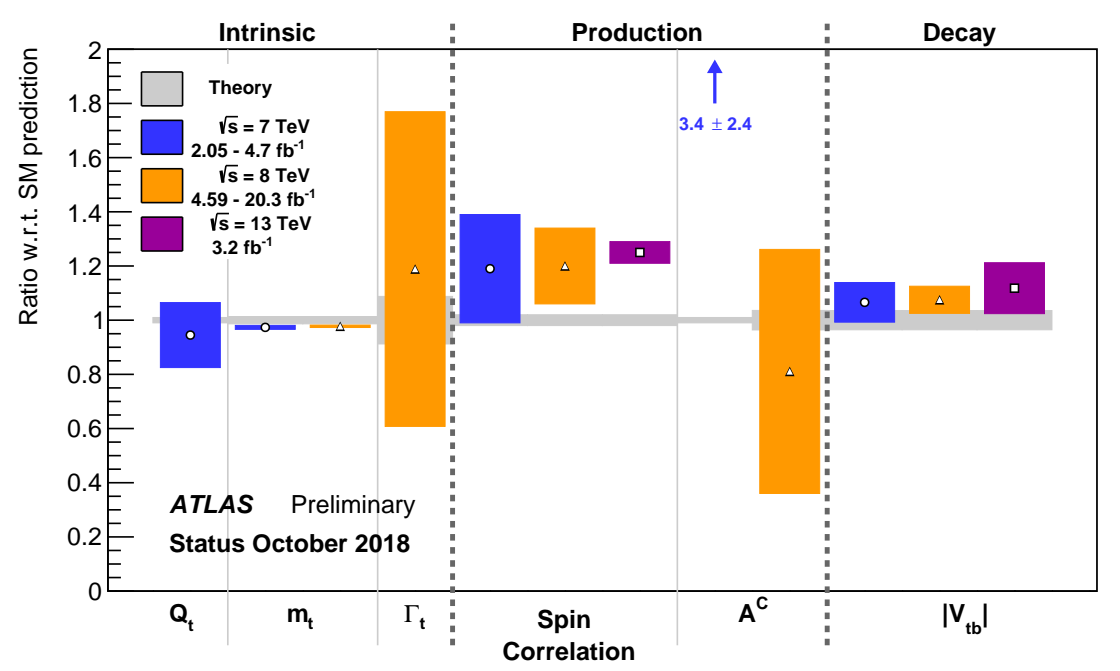

Figure 4: Overview of top-quark properties measurement by the ATLAS collaboration [3].

The two most recent top-quark properties measurements released by the ATLAS collaboration have been presented. The normalised differential cross section, $\mathcal{R}$, of top-quark pair production in association with an energetic jet is presented as a function of the inverse of the invariant mass of the $t \bar{t}+1$-jet system $\rho_{\mathrm{s}}=2 m_{0} / m_{t \bar{t}+1 \text {-jet }}$. The measurement is performed using $p p$ collision data at a centre-of-mass energy of $8 \mathrm{TeV}$ collected by the ATLAS experiment at the LHC in 2012. The data sample corresponds to an integrated luminosity of $20.2 \mathrm{fb}^{-1}$. The distribution of $\mathcal{R}$ observed in the semileptonic final state is unfolded to the parton and particle levels. The result from data unfolded to parton level is compared with the NLO QCD predictions in two different renormalisation schemes. The top-quark mass extracted in the pole-mass scheme yields $m_{t}^{\text {pole }}=171.1 \pm 0.4$ (stat.) \pm 0.9 (syst. $)_{-0.3}^{+0.7}$ (theo.) $\mathrm{GeV}$. The result for the running mass in the $\overline{\mathrm{MS}}$ scheme is $m_{t}\left(m_{t}\right)=162.9 \pm 0.5$ (stat. $) \pm 1.0$ (syst. $)_{-1.2}^{+2.1}$ (theo.) $\mathrm{GeV}$. The result for $m_{t}\left(m_{t}\right)$ suffers from a larger theoretical uncertainty as compared with the pole mass. This is due to a larger dependence on the renormalisation and factorisation scales of the $\overline{\mathrm{MS}}$ scheme in the most sensitive region close to the $t \bar{t}+1$-jet threshold.

Absolute and normalised differential $t \bar{t}$ cross-sections have been measured as a function of $\Delta \phi$ and $\Delta \eta$, between the two charged leptons in the $e \mu$ decay channel using $13 \mathrm{TeV}$ data collected in 2015 and 2016. The $\Delta \phi$ differential cross-section is also measured as a function of the $t \bar{t}$ invariant mass. None of the studied generators are able to reproduce the normalised $\Delta \phi$ distribution. A comparison was made with fixed-order predictions at NNLO in QCD and in an expansion at NLO in QCD and EW couplings with a fixed scale choice, with the former slightly improving the description of the data, and the latter describing the data but with large scale uncertainties. An extraction of spin correlation was performed using the normalised parton-level $\Delta \phi$ observable. The spin correlation was found to be higher than that predicted by the SM as implemented in NLO MC generators with a significance of $3.2 \sigma$. However, the measurement agrees well with the prediction by the expansion at NLO in QCD and EW couplings. 


\section{Acknowledgements}

The author would like to thank René Poncelet, Markus Schulze and Miriam Watson for useful discussions. This work was partially funded by the European Research Council under the European Union's Seventh Framework Programme ERC Consolidator Grant Agreement n. 617185 (TopCoup) and by the German Federal Ministry of Education and Research (BMBF) in FSP-103 under grant n. 05H15PDCAA.

\section{References}

[1] ATLAS Collaboration, Measurement of the top-quark mass in t $\bar{t}+1$-jet events collected with the ATLAS detector in pp collisions at $\sqrt{s}=8 \mathrm{TeV}$, submitted to JHEP, arXiv:1905.02302 [hep-ex].

[2] ATLAS Collaboration, Measurement of the top quark mass in the $t \bar{t} \rightarrow$ lepton+jets channel from $\sqrt{s}=8$ TeV ATLAS data and combination with previous results, Eur. Phys. J. C79 (2019) 290, arXiv:1810.01772 [hep-ex].

[3] ATLAS Collaboration, Top Working Group Summary Plots - Autumn 2018, ATL-PHYS-PUB-2018-034, CERN, 2018, cds.cern.ch/record/2647993.

[4] ATLAS Collaboration, Measurements of top-quark pair spin correlations in the e $\mu$ channel at $\sqrt{s}=13$ TeV using pp collisions in the ATLAS detector, submitted to Eur. Phys. J. C, arXiv:1903.07570 [hep-ex].

[5] A. Behring, M. Czakon, A. Mitov, A. S. Papanastasiou and R. Poncelet, Higher order corrections to spin correlations in top quark pair production at the LHC, arXiv:1901.05407 [hep-ph].

[6] W. Bernreuther, D. Heisler and Z. G. Si, A set of top quark spin correlation and polarization observables for the LHC: Standard Model predictions and new physics contributions, JHEP 12 (2015) 026, arXiv:1508.05271 [hep-ph]. 